\title{
MEJORA DEL CLIMA DE CONVIVENCIA EN EL AULA MEDIANTE UNA METODOLOGÍA DE INVESTIGACIÓN - ACCIÓN
}

Cárdenas Reyes, Juan Luis (juanlcr@correo.ugr.es); Lara Guardia, Gracia Nacarí (nacarilg21@correo.ugr.es), Molina Arias, Cristina (kmolina@correo.ugr.es); Moreno Lucena, Álvaro (amlpl@correo.ugr.es); Morilla Navarro, Verónica (vmn90@correo.ugr.es). Universidad de Granada

Supervisado por: Puri Slamerón Vílchez. Departamento de Método de investigación y Diagnóstico en Educación. Universidad de Granada

Fecha de recepción: 22 de abril de 2014.

Fecha de revisión: 30 de abril de 2014.

Fecha de aceptación: 11 de mayo de 2014.

RESUMEN: El propósito de la investigación fue la elaboración de una escala para evaluar la convivencia en el aula mediante una metodología de Investigación-Acción. Se seleccionó una muestra de 21 alumnos pertenecientes al tercer curso de Educación Secundaria Obligatoria del Instituto Ángel Ganivet de la provincia de Granada en el año académico 2013 - 2014. La metodología utilizada fue la de Investigación-Acción, ya que a través de ésta se estudia una situación social concreta con vistas a analizarla y mejorarla. A su vez, la temática elegida fue la convivencia, debido a que es considerada una de las claves para mejorar la calidad de la enseñanza y de la escuela en su globalidad. Los estudiantes diseñaron una escala para evaluar la propia convivencia en el aula, en colaboración con los investigadores, la cual fue posteriormente autoadministrada para detectar las distintas problemáticas y proponer mejoras para solventarlas. Los resultados, en líneas generales, nos muestran que existe una buena convivencia en este grupo. Sin embargo, se ha detectado que los problemas que influyen en una peor convivencia son los relacionados con la interacción profesor-alumno.

Palabras clave: Trabajo de investigación, interacción social, relación entre pares, relación profesor-alumno.

ABSTRACT: The purpose of this research was to investigate the elaboration of a scale to evaluate the classroom co-existence by means of an Action-Research methodology. Therefore, a sample of 21 students belonging to third grade of Ángel Ganivet Secondary School of the province of Granada, in the 2013 - 2014 Academic Year we used for this research. The methodology which was used was Action-Research since one of the aims of the study was for specific social situation situations with the aim of analysing and improving it. The chosen topic for the study was classroom co-existence due to the fact that it is considered as one of the keys to improve the teaching quality and education in general. Students designed a scale to evaluate their own classroom co-existence with the collaboration of investigators which was auto administered later to try to detect the different types of problems and to propose some improvements to try to solve them. The results, in general lines, show us that a good classroom coexistence exists in the group in question. However, it was detected that the problems which influence in worse classroom co-existences are related to teacher-student interaction.

Keywords: Research work, social interaction, peer relationship, student teacher relationship. 


\section{INTRODUCCIÓN}

Actualmente, son muchas las definiciones que existen del término convivencia. Algunos autores como Teixidó y Castillo (2013), definen este concepto como el arte de vivir juntos y de compartir la vida con el resto de personas que nos rodean, en nuestro día a día. Por su parte, Rodríguez (2011) lo define como aquel proceso que consiste en compartir la vida con los otros, aprendiendo de lo propio y de lo que éstos nos ofrecen.

Fernández (2010) defiende que la convivencia ha pasado de ser una cuestión requerida por aquellos centros educativos que estaban interesados por esta temática y la querían incorporar dentro de su línea de trabajo, a ser un objetivo prioritario de todos los centros educativos. De tal forma, que si no existen buenas relaciones de convivencia y respeto en los centros escolares, las tareas de enseñanza y aprendizaje se convierten en unas prácticas de difícil realización en los mismos y con unos resultados pocos exitosos. Este mismo autor afirma que "la convivencia pasa de ser un tema periférico a los procesos de enseñanza y al desarrollo democrático de la vida en el aula, a considerarse un paso previo para conseguir la calidad de la enseñanza y de la escuela en su conjunto".

Teixidó y Castillo (2013) consideran que la convivencia ha adquirido, actualmente, un papel muy importante dentro de los centros educativos, como consecuencia de los elevados comportamientos y niveles de violencia que se han generado en torno a los mismos. Dichos problemas, se caracterizan según Rodríguez (2011) por desarrollarse u originarse dentro del ámbito educativo bien por los propios estudiantes, por éstos y los profesores, por estos últimos y las familias, por la organización de la escuela o por el desarrollo de las tareas diarias, entre otras cuestiones importantes. Martínez (2001), asegura que en todas las relaciones de convivencia pueden surgir una serie de conflictos o enfrentamientos de diversa índole entre los individuos, que podrian llegar a debilitar o romper su propia relación. Esto se origina porque, en determinadas ocasiones, los desacuerdos existentes entre las personas pueden derivar en un clima de conflicto que hace desaparecer la convivencia entre los individuos.

El informe sobre la convivencia de los centros educativos (2006) defiende que los tipos de conductas más características que alteran la adecuada convivencia dentro de los centros educativos son la disrupción en las aulas, como por ejemplo los problemas de disciplina y de orden, los comportamientos disruptivos injustificados o la falta de atención y motivación; los problemas de disciplina entre los profesores y los estudiantes, como las agresiones verbales e insultos o las faltas de respeto, entre otras; el maltrato entre los compañeros (bullying); el acoso sexual y la discriminación por orientación sexual; el absentismo y/o deserción escolar; y las prácticas ilegales (plagio en los trabajos, copiar en los exámenes, etc.).

Este mismo informe diferencia dos tipos de respuestas educativas para abordar la convivencia que se produce en los colegios e institutos, unas de tipo global y otras más especializadas. Las primeras se caracterizan por abordar la convivencia como una cuestión del propio centro, con la finalidad de prevenir y solucionar los problemas de comportamiento existentes desde el propio contexto educativo; y las segundas porque afrontan la convivencia desde una cuestión más específica, mediante la cual se plantean unos programas que tratan de hacer frente a los comportamientos antisociales que se producen dentro del centro.

De la misma forma, Fernández (1999) defiende la existencia de varias formas de actuación necesarias para abordar y dar respuesta a los problemas de convivencia que surgen entre los individuos. De manera que destaca la concienciación y el 
reconocimiento de problemas para ser capaces de establecer unas normas compartidas. Es por ello que la Investigación-Acción parece ser una respuesta adecuada para abordar esta temática dentro del contexto escolar, siendo ésta la base de una construcción colaborativa del conocimiento adecuada a un contexto concreto (Casals, Vilar, Ayats, 2008) y una metodología útil para el autodiagnóstico y aprendizaje de estrategias de desarrollo (Alguacid, Basagoiti y Camacho, 2006). La Investigación-Acción rompe, por tanto, la tradicional separación entre investigadoresinvestigados, interviniendo en el objeto de estudio, diseño, diagnóstico, formulación de propuestas y evaluación (Alguacid, Basagoiti y Camacho, 2006) y poniendo en juego el reconocimiento, desestructuración y reestructuración del saber, mediante la confrontación de las distintas opiniones de los participantes (Del Gobbo, 2009).

En cuanto a esta metodología, no existe una definición concreta y consensuada (Colas-Bravo, 1998). No obstante autores como Elliot (2005), la definen como "el estudio de una situación social con miras a mejorar la calidad de la acción dentro de ella" con el fin de optimizar y/o solucionar problemas cotidianos, mediante una investigación autorreflexiva de los mismos. Otros autores como Romera-Iruela (2011) definen la Investigación-Acción como una vinculación entre la teoría y la práctica permitiendo el avance y la mejora permanente de ésta. Siguiendo esta línea, Casals, Vilar y Ayats (2008) explican ésta como una actividad promovida por un gran grupo que tiene como objetivo transformar sus circunstancias basándose en una concepción compartida por todos sus miembros.

Mackernan (2001), fundamenta la importancia de la Investigación-Acción en tres aspectos principales: los participantes de la investigación, son quienes mejor entienden el problema al encontrarse inmersos en el; las conductas están influenciadas en su mayor parte por el contexto en el cual se desarrollan; y una de las mejores formas de analizar la realidad, es mediante el uso de metodologías cualitativas. Así la Investigación-Acción permite a sus participantes reflexionar sobre su práctica diaria, detectando y analizando posibles conductas conflictivas con el objeto de buscar soluciones y tomar decisiones conjuntamente, favoreciendo así un aumento de la responsabilidad individual y grupal (Pérez, Amador \& Vargas, 2011). Además, contribuye a mejorar y cambiar la práctica del currículum, debido a que los profesionales se benefician de los resultados de sus propias investigaciones (RomeraIruela, 2011).

\section{MÉTODO}

Este estudio se realizó durante el curso académico 2013/2014 dentro del Instituto Público Ángel Ganivet de la provincia de Granada, con el objetivo de analizar cómo los alumnos y alumnas de Educación Secundaria Obligatoria eran capaces de poner en funcionamiento, por ellos mismos, un tipo de metodología denominada InvestigaciónAcción, por medio del análisis y evaluación del propio estado de convivencia escolar existente dentro de su centro.

\section{Participantes}

En este estudio han participado voluntariamente 20 estudiantes (13 alumnos y 7 alumnas) de $3^{\circ}$ de Educación Secundaria Obligatoria del Instituto Ángel Ganivet de la provincia de Granada, con una edad comprendida entre los 14 y los 15 años. Estos estudiantes tienen una procedencia diversa, de forma que la mayoría residen en el cinturón metropolitano de la ciudad, como los pueblos de Peligros, Víznar, Deifontes u otros de la Vega de Granada. Aunque un gran número de ellos procede de barrios 
bastante cercanos como el Albaicín. De forma relativamente general, el nivel socioeconómico de las familias de este alumnado es medio-alto, ya que la mayoría de los progenitores suele tener estudios superiores y un trabajo que les ofrece estabilidad.

\section{Instrumentos}

Para orientar a los alumnos/as de tercero de Educación Secundaria Obligatoria, en el desarrollo de una metodología de Investigación-Acción, se ha utilizado la Escala de Clima Social de Moos, Moos y Trickett (1995), y la Escala de Convivencia en el Aula realizada por Polo, León y Gozalo (2013), como una adaptación de la escala anterior, por medio de las cuales se elaboró un cuestionario de preguntas para analizar el estado de convivencia del grupo - clase.

La escala de clima social de Moos, Moos y Trickett diseñada para estudiar el propio clima social de la clase, las relaciones entre profesor-alumno y alumno-alumno, y la estructura organizativa del aula, consta de 90 ítems a los que se ha de responder con una doble alternativa (verdadero / falso). Estos ítems miden nueve subescalas diferentes comprendidas en cuatro dimensiones: la dimensión relacional que mide el grado en el que los estudiantes están integrados en el aula, así como las relaciones existentes entre los mismos; la de desarrollo personal o de autorrealización que se centra en la realización de las diversas tareas escolares y de las propias asignaturas; la de estabilidad o sistema de mantenimiento que se basan en el desarrollo del funcionamiento de la clase, la organización, control, etc.; y la del cambio que evalúa el grado en que hay diversidad e innovación en las tareas de clase. En cada uno de los ítems que recoge esta escala se otorga un punto por cada respuesta que coincida con la clave administrada por la prueba, por lo que la puntuación oscila entre 0 y 10 , siendo 0 considerado un bajo nivel, y 10 un elevado nivel.

Por su parte la escala de clima de convivencia en el aula (Polo, León y Gozalo, 2013) diseñada para medir el propio clima de convivencia entre los compañeros/as de clase y el profesor, consta de 43 ítems, y se presenta en formato Likert con cinco intervalos en forma numérica de 1 al 5 , que representan un continuo que va desde 1 que significa "Nada" y 5 que significa "Mucho".

Por otro lado, la escala construida mediante la metodología de Investigación-Acción con el grupo de alumnos, ha sido llamada "Escala para evaluar la convivencia en el aula" (véase en anexo 1). Esta consta de 45 preguntas y mide 5 subescalas, las cuales abarcan cinco dimensiones correspondientes a: aspectos personales, aspectos relacionados con la sexualidad, convivencia en relación a las normas/organización escolar, convivencia en relación al alumnado y convivencia en relación al profesorado. Esta escala se presenta en formato Likert, concretamente con cinco intervalos numéricos del 1 al 5 , representando un continuo que va desde 1 que significa "Totalmente en desacuerdo" y 5 que significa "Totalmente de acuerdo". No obstante, se presentan preguntas en las que su valor es inverso como son los ítems 5, 6, 8, 9, $14,25,29,30,31,32,42$ y 45.

\section{Procedimiento}

Para la realización de la presente investigación se han llevado a cabo tres sesiones, durante las horas de tutoría de $3^{\circ}$ de ESO, las cuales se impartían los viernes a cuarta hora.

En la primera sesión realizada el día 18 de abril de 2014, se realizó una breve exposición teórica acerca de la metodología de Investigación-Acción y se abordó la temática de la convivencia escolar, extrayendo todos los factores que influían en la misma. Tras esto, se dividió la clase de forma heterogénea en cuatro grupos de 
trabajo, los cuales tenían que elaborar una escala para evaluar la convivencia basándose en los factores que se habían extraído previamente (véase anexo 2). Para el asesoramiento llevado a cabo en la elaboración de las distintas escalas, se tomó como referencia la Escala de Clima Social de Moos, Moos y Trickett (1995) y la Escala de Clima de Convivencia en el Aula de Polo, León y Gozalo (2013), previamente explicadas en el apartado instrumentos.

En la segunda sesión que se realizó el 25 de abril de 2014, los alumnos cumplimentaron la escala que los investigadores elaboramos a partir de las cuatro escalas diseñadas por los grupos en clase. Esta escala tuvo en cuenta aquellos ítems que no se repetían y que evaluaban la convivencia en el aula de un grupo. Los alumnos rellenaron la escala y posteriormente se realizó un debate a nivel grupal, en el que los alumnos reflejaron las reflexiones que habían realizado al contestar a las distintas preguntas de la escala.

Finalmente, en la tercera sesión correspondiente al día 9 de mayo de 2014, los investigadores comunicaron los resultados de las escalas cumplimentadas por los alumnos y se realizó un análisis de aquellas problemáticas que detectaban en cuanto a la convivencia en su grupo Posteriormente, se les animó a proponer una serie de mejoras para solventar dichas problemáticas. Para concluir, se realizó un debate y una puesta en común a nivel de gran grupo sobre aquellas deficiencias detectadas así como las mejoras propuestas.

\section{RESULTADOS}

Una vez diseñado el instrumento propio para la evaluación de la convivencia en esta aula y tras su auto-administración al presente grupo clase, se obtuvieron los siguientes resultados en relación a las diferentes dimensiones que componen nuestro estudio (figura 1).

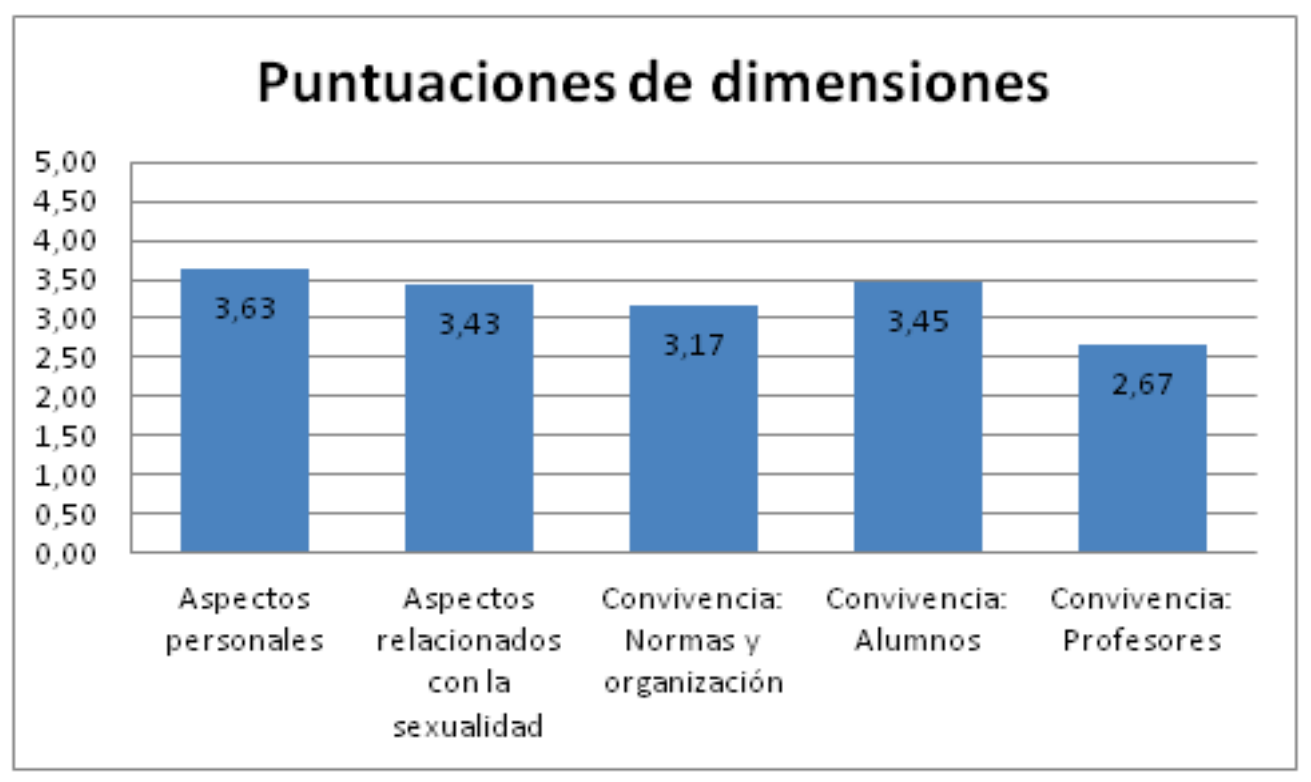

Figura 1: Media obtenida por dimensión de la escala para evaluar la convivencia en el aula. Fuente: Elaboración propia.

La figura 1 nos revela cómo las puntuaciones obtenidas en cada una de las dimensiones se encuentran por encima de 2,5, lo cual indica que en esta aula no 
existen problemas graves de convivencia. Continuando con el análisis de esta figura podemos comprobar cómo la dimensión en la cual se ha obtenido una puntuación más baja $(2,67)$ es la que guarda relación con la convivencia entre profesorado y alumnado. Por el contrario, la dimensión la cual ha obtenido una mayor puntuación es la relativa a los aspectos personales $(3,63)$ que favorecen una mejor convivencia dentro del aula.

Una vez analizados estos datos, se ha procedido al estudio de la escala para evaluar la convivencia en el aula por ítem (figura 2).

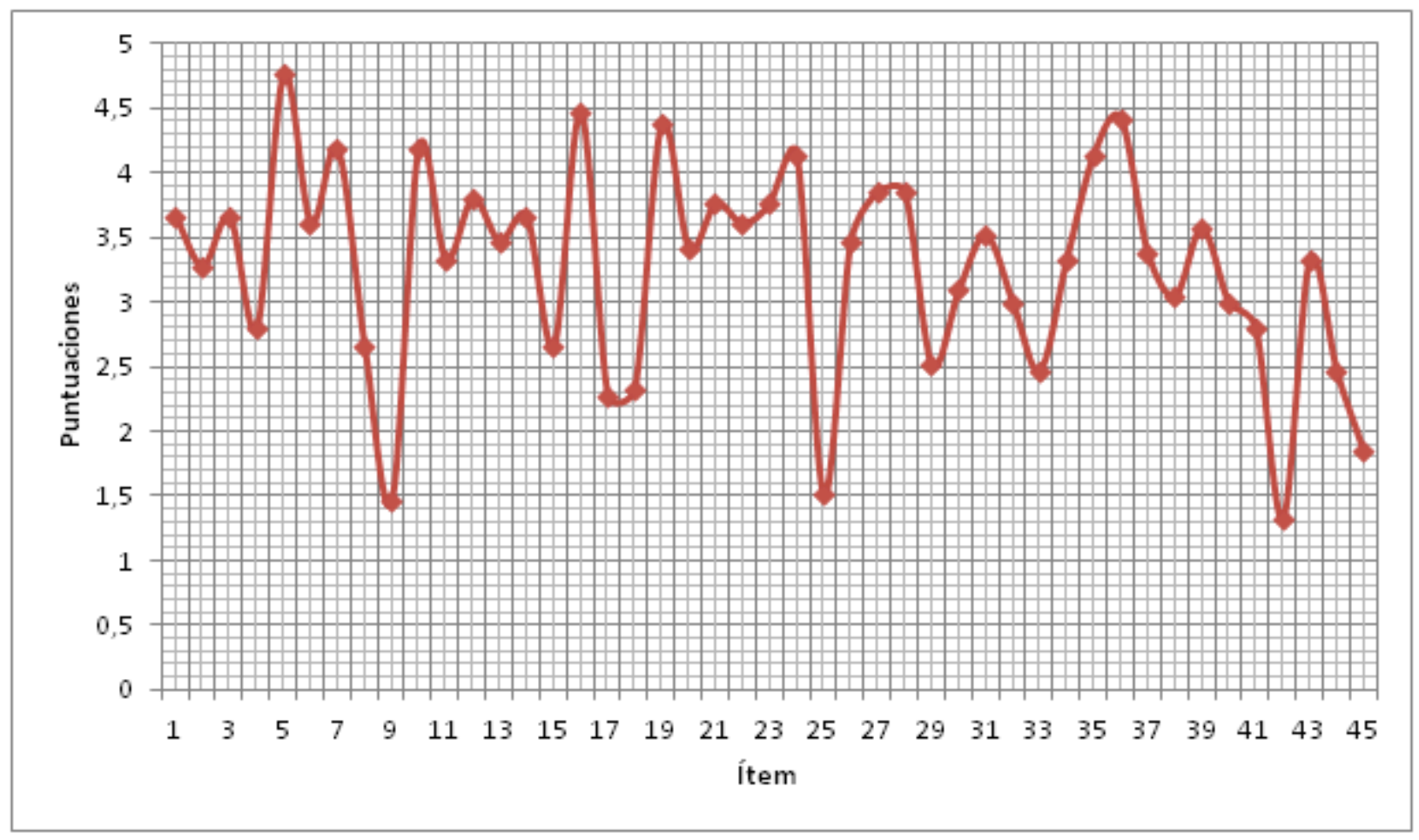

Figura 2: Media obtenida por ítem de la escala para evaluar la convivencia en el aula.

Fuente: Elaboración propia

La figura 2 se observa una distribución de las respuestas medias obtenidas por los estudiantes en cada uno de los ítems que componen esta escala. Los resultados nos muestran que los ítems con mayor puntuación obtenida y por tanto inciden en una mejor convivencia en el aula son los ítems $5(4,76), 7(4,19), 10(4,19), 16(4,47), 19$ $(4,38), 24(4,14), 35(4,14)$ y $36(4,42)$. Por lo cual, se deduce que estos estudiantes no muestran enfado cuando algún compañero saca más nota que ellos, respetando asimismo las distintas opiniones, ideas y orientaciones sexuales de sus compañeros. Además, podemos comprobar cómo estos alumnos presentan un buen conocimiento de las normas existentes en clase, asistiendo de forma puntual y continua a clase, y mostrando un sentido amplio de la justicia escolar. A su vez, los datos revelan la existencia de una buena relación entre los compañeros tanto en clase como en el recreo.

Por otro lado, los resultados obtenidos en la figura 2 nos informan de aquellos ítems que influyen negativamente en la convivencia dentro del aula, siendo estos los ítems 9 $(1,47), 17(2,28), 18(2,33), 25(1,56), 29(2,52), 33(2,47), 42(1,33), 44(2,47)$ у 45 $(1,85)$. Tras la detección de los puntos débiles que afectan a la convivencia de este grupo clase, los alumnos propusieron una serie de medidas con el objetivo de mejorar dicha convivencia (Tabla 1). 
Tabla 1. Propuestas por el alumnado para la mejora de la convivencia en el aula

\begin{tabular}{cc} 
Ítem & \multicolumn{1}{c}{ Problema } \\
9 & Cuando el/la profesor/a no está en clase \\
& se crea mucho alboroto en el aula.
\end{tabular}

Solución

"Debemos concienciarnos de que debemos estar más en silencio y ser más civilizados. Para ello, proponemos que haya siempre un profesor en el aula que nos controlen".

"Una buena solución sería que nos dieran recompensas (más tiempo en el patio, excursiones, actividades libres dentro del aula, etc.) cada vez que no hacemos ruido y nuestro comportamiento es correcto".

"Las clase son muy aburridas porque el profesor siempre explica los primeros minutos y luego manda muchos ejercicios. No la liaríamos tanto si las actividades de clase fueran más interesantes y entretenidas".

\begin{tabular}{|c|c|c|}
\hline 17 & $\begin{array}{l}\text { En las zonas comunes (pasillo, salón de } \\
\text { actos, etc.) existe orden. }\end{array}$ & $\begin{array}{l}\text { "Que haya más profesores en los cambios de clase" } \\
\text { "Evitar que haya tantos cambios de aula para que así no haya alboroto en los } \\
\text { pasillos en los intercambios" } \\
\text { "Que haya un encargado de aula que mantenga el orden entre los compañeros" }\end{array}$ \\
\hline 18 & $\begin{array}{l}\text { Las partes escolares son útiles para } \\
\text { mejorar la convivencia. }\end{array}$ & $\begin{array}{l}\text { "Que toda la clase conociera los motivos por los que se castiga o se abre un parte" } \\
\text { "Que no se repartieran los partes como chicles" } \\
\text { "Que solo se pongan partes cuando existan motivos lo suficientemente graves". }\end{array}$ \\
\hline 25 & $\begin{array}{l}\text { Existen pequeños subgrupos dentro de la } \\
\text { clase. }\end{array}$ & $\begin{array}{l}\text { "Haciendo más cosas dentro del propio instituto que nos unieran, como por ejemplo } \\
\text { talleres de cocina, deportes, etc." } \\
\text { "Que por las tardes se organizaran actividades como, por ejemplo, gymkanas, } \\
\text { campeonatos de fútbol, etc." } \\
\text { "Que se hicieran más actividades para conocerse, relacionarse, etc., de forma que } \\
\text { no siempre nos relacionarnos con las mismos, sino que pudiéramos conocer más } \\
\text { profundamente a todos los compañeros" }\end{array}$ \\
\hline 29 & $\begin{array}{l}\text { Existen motes ofensivos que afectan a la } \\
\text { relación con mis compañeros. }\end{array}$ & $\begin{array}{l}\text { "Preguntarle a esa persona si no le gusta el mote que tiene y en caso de que diga } \\
\text { que sí evitar decírselo" } \\
\text { "Hacer actividades poniéndose en el lugar del otro" } \\
\text { "Solo decírselo a la gente a los que no se lo toman en serio" } \\
\text { "Que el profesor intervenga parándolo y si no hacen caso poner negativos" } \\
\text { "Intentar llevarse bien, o si no, al menos pasar el uno del otro" }\end{array}$ \\
\hline 33 & $\begin{array}{l}\text { Considero que el delegado es una buena } \\
\text { representación del grupo-clase. }\end{array}$ & $\begin{array}{l}\text { "Una solución seria que las personas que quieran presentarse como delegado } \\
\text { hicieran una especie de campaña electoral, donde dijeran a qué se comprometen a } \\
\text { hacer con la clase" } \\
\text { "Seleccionar a esa persona con conciencia y responsabilidad y no con bromas y } \\
\text { cachondeos" } \\
\text { "Que en el caso de que el delegado de clase no cumpla bien su función, se pueda } \\
\text { cambiar a mitad de curso". }\end{array}$ \\
\hline 42 & $\begin{array}{l}\text { Existe favoritismo del profesorado con } \\
\text { determinados alumnos que afecta a la } \\
\text { relación con mis compañeros. }\end{array}$ & $\begin{array}{l}\text { "Que los profesores traten a todos los alumnos por igual, sin favorecer a unos sobre } \\
\text { otros. La mejor solución sería hablar con el profesor y comentarle esta situación } \\
\text { para que sepan nuestra opinión y traten remediarla". } \\
\text { "No creo que podamos solucionar algo que solo ellos pueden arreglar, ya que es } \\
\text { algo mental. Ellos siempre van a tener a un favorito. Tampoco, estamos pidiendo } \\
\text { que sean robots pero sí que intenten dejar de lado sus preferencias y } \\
\text { sentimientos". } \\
\text { "Los profesores deberían ser más conscientes de cómo es cada uno, para ello } \\
\text { creemos que sería buena idea hacer act. de convivencia donde participemos todos } \\
\text { los estudiantes y sus profesores (día de campo, juegos para conocernos mejor } \\
\text { unos a otros, comidas, celebrar los cumpleaños de la clase, etc.)." }\end{array}$ \\
\hline 44 & $\begin{array}{l}\text { El profesorado da ejemplo sobre las } \\
\text { normas establecidas en clase. }\end{array}$ & $\begin{array}{l}\text { "Los profesores deberían tener normas, no solo nosotros y cuando algún profesor } \\
\text { no cumpla con las normas establecidas ponerle alguna amonestación". } \\
\text { "Que los profesores den ejemplo cumpliendo también con las normas que ellos } \\
\text { mismos nos ponen". } \\
\text { "Cuando algún profesor utilice el móvil en clase, decírselo al director para que } \\
\text { tomen medidas". } \\
\text { "Poner una hucha en clase y cada vez que alguien (profesor y alumno) no cumpla } \\
\text { alguna norma echar } 1 € \text { Al final de curso con el dinero recaudado irnos de } \\
\text { excursión todos". }\end{array}$ \\
\hline 45 & Existen profesores que no son permisivos. & $\begin{array}{l}\text { "Intentar convencerlos para que sean menos estrictos, ya que nos falta diálogo". } \\
\text { "Mejorar la comunicación entre profesor-alumno haciendo asambleas semanales". } \\
\text { "Que los profesores muestran mayor interés por las preocupaciones y gustos de } \\
\text { sus estudiantes". }\end{array}$ \\
\hline
\end{tabular}

\section{DISCUSIÓN}

La investigación realizada, nos ha proporcionado la oportunidad de aprender cómo el proceso de Investigación-Acción es un recurso muy eficaz dentro de los centros educativos, no solo para mejorar su práctica diaria y los problemas en ellos existentes, sino también ofreciendo la oportunidad tanto a alumnos como profesores de 
reflexionar de forma autónoma sobre sus propios problemas con el fin de mejorarlos y enriquecerse conjuntamente.

Desde el punto de vista del alumnado, ha sido una actividad que ha beneficiado al grupo, puesto que ha permitido que éstos reflexionen de forma conjunta sobre su propia convivencia dentro del colegio y que mejoren la relación entre ellos presente.

Una de las grandes limitaciones que hemos encontrado a lo largo de nuestro trabajo ha sido el poco tiempo disponible para desempeñar este tipo de metodología en el aula, lo cual pensamos que ha podido dificultar el proceso de reflexión y asimilación que requería este tipo de investigación. Para futuras investigaciones, se podría proponer que el profesorado participara en esta investigación, puesto que así podríamos obtener unos resultados más significativos de cómo es la convivencia en el centro educativo, no solo a nivel de alumnado, sino también de profesor - alumno.

\section{REFERENCIAS BIBLIOGRÁFICAS}

Alguacid J., Basagoiti M. y Camacho J. (2006). Investigación-acción participativa en el barrio de San Cristóbal de los Ángeles (distrito de Villaverde, Madrid). Cuadernos de Trabajo Social. 19, 331-346.

Casals, Vilar \& Ayats (2008). La investigación-acción colaborativa: reflexiones metodológicas a partir de su aplicación en un proyecto de música y lengua. Revista Electrónica Complutense de Investigación en Educación Musical, 5 (4).

Colas, M. (1998). Métodos y técnicas cualitativas de investigación en psicopedagogía. En: Colás, M; Buendía, L; Hernández, F (eds.) (1998) Métodos de investigación en psicopedagogía. Madrid, España: Editorial MC Graw Hill Latinoamericana.

Del Gobbo, G. (2009). La investigación acción participativa entre formación y desarrollo. Recuperado de http://goo.gl/0SY5JT

Elliott, J. La investigación - acción en educación (5ª ed.). Madrid: Morata.

Fernández, I. (1999). Prevención de la violencia y resolución de conflictos. El clima escolar como facto de la calidad. Madrid: Narcea.

Fernández, I. (2010). Evolución de la convivencia en los centros escolares. Gázquez, J. J., y Pérez, M. C. (Coord.), La convivencia escolar. Aspectos psicológicos y educativos. Buenos Aires: GEU., 65-68.

Consejo Escolar de Andalucía. (2006). Informe sobre la convivencia en los centros educativos. Recuperado de http://goo.gl/6gtTgd

Mackernan, J. (2001). Investigación y currículum (6ª ed.). Madrid: Morata.

Martínez, V. (2001). Convivencia escolar: problemas y soluciones, Revista Complutense de Educación, 12 (1), 295-318.

Moos, R., Moos, B., \& Trickett, E. (1995). Escalas de clima social. España: TEA.

Pérez, Amador \& Vargas (2011). Resolución de conflictos en las aulas: un análisis desde la investigación-acción. Pedagogía social. Revista interuniversitaria 18, 99-114. 
Polo, M., León, B. y Gozalo, M. (2013). Perfiles de la dinámica bullying y clima de convivencia en el aula. Apuntes de Psicología, 31(2), 135-144.

Rodríguez, P. L. (2011). Análisis de la convivencia escolar en aulas de educación primaria, Revista Iberoamericana de Educación, 55 (3).

Romera-Iruela (2011). La investigación-acción en la formación del profesorado. Revista Española de Documentación Científica, 34 (4), 597-614. doi: 10.3989/redc.2011.4.836.

Teixidó y Castillo (2013). Prácticas de mejora de la convivencia escolar. Recopilación, sistematización y análisis de buenas prácticas. Málaga: Ediciones Aljibe.

Torres, M. T. (2010). El problema de la violencia entre universitarios abordado desde el enfoque de la Investigación - Participación - Acción. Investigación Universitaria Multidisciplinaria, 9(9), 27-36. 


\section{ANEXO 1. ESCALA PARA EVALUAR LA CONVIVENCIA EN EL AULA}

\section{ESCALA PARA EVALUAR LA CONVIVENCIA EN EL AULA}

A continuación te encontrarás con 45 preguntas en relación a la convivencia con tu grupo-clase. Contesta sinceramente a cada una de ellas, en relación si te encuentras de acuerdo o no con la afirmación que se plantea. Siendo $1=$ Totalmente en desacuerdo, 2= En desacuerdo, 3= Ni de acuerdo ni en desacuerdo, $4=$ De acuerdo y $5=$ Totalmente de acuerdo. Te recordamos que la escala es totalmente anónima y confidencial.

\section{EDAD:}

SEXO: CURSO:

Me siento cómodo/a cuando hablo en público.

Mi motivación en clase es alta.

Realizo siempre mis deberes.

En general, las asignaturas que curso me resultan interesantes.

Me enfado cuando algún compañero saca mejor nota que yo.

Me disgusta que me confundan con alguno de mis compañeros.

En clase se respetan las orientaciones sexuales de todos los compañeros.

El tener pareja en clase afecta negativamente a la convivencia del grupo.

Cuando el/la profesor/a no está en clase se crea mucho alboroto en

el aula.

Conozco las normas de la clase.

Cumplo las normas de la clase.

Los alumnos/as hemos participado en la creación de las normas de clase.

Existe orden y organización en clase (delegado, acuerdos profesoralumnos, etc.).

Suelo copiar en los exámenes o tareas.

Nos mantenemos en silencio cuando trabajamos en la biblioteca del centro.

Asisto a clase de manera continua y puntual.

En las zonas comunes (pasillo, salón de actos, etc.) existe orden.

Los partes escolares son útiles para mejorar la convivencia.

Existe una buena relación con mis compañeros.

Confío en mis compañeros.

Normalmente soluciono los problemas con mis compañeros de forma dialogante.

No existe discriminación en el aula.

En clase nos ayudamos mutuamente.

Existe un buen ambiente en el recreo con los compañeros.

Existen pequeños subgrupos dentro de la clase.

Tengo contacto con mis compañeros fuera del horario escolar.

El grupo está abierto a la integración de los alumnos nuevos.

Se respetan las distintas orientaciones religiosas.

Existen motes ofensivos que afectan a la relación con mis compañeros.

Existen peleas en clase que afectan a la convivencia con mis compañeros.

Surgen conflictos con mis compañeros a través de las redes sociales. (Whatsapp, Tuenti, Twitter, Facebook, etc.)

Siento que alguno de mis compañeros me critica a la espalda.

Considero que el delegado es una buena representación del grupoclase.

Respeto el turno de palabra con mis compañeros.

Respeto las distintas ideas de mis compañeros en la realización de trabajos grupales.

Considero injusto el castigo colectivo en caso de haber un único responsable.

Tengo una buena relación con el profesorado

Generalmente el profesorado confía en mí.

El profesorado me suele respetar.

Cuando presto el material me lo devuelven en buen estado.

El profesorado suele mostrar interés dedicando tiempo a hablar con cada uno de los alumnos en caso de necesidad.

Existe favoritismo del profesorado con determinados alumnos que afecta a la relación con mis compañeros.

Suelo llegar fácilmente a un acuerdo con los profesores.

El profesorado da ejemplo sobre las normas establecidas en clase.

Existen profesores que no son permisivos.

\begin{tabular}{l|l|l|l}
\multicolumn{5}{c}{ CURSO: } \\
\hline & 1 & 2 & \\
\hline & 1 & 2 & \\
\hline & 1 & 2 & \\
\hline & 1 & 2 & \\
\hline los & 1 & 2 & \\
\hline & 1 & 2 & \\
\hline del & 1 & 2 & \\
\hline
\end{tabular}

\begin{tabular}{|l|l|l|}
\hline 3 & 4 & 5 \\
\hline 3 & 4 & 5 \\
\hline 3 & 4 & 5 \\
\hline 3 & 4 & 5 \\
\hline 3 & 4 & 5 \\
\hline 3 & 4 & 5 \\
\hline 3 & 4 & 5 \\
\hline
\end{tabular}

1

2

$2 \quad 3$

\begin{tabular}{|c|}
\hline 1 \\
\hline 1 \\
\hline 1 \\
\hline
\end{tabular}

\begin{tabular}{|l|l}
1 & \\
\hline 1 & 2 \\
\hline 1
\end{tabular}

\begin{tabular}{l|l}
\hline 2 & 3
\end{tabular}

\begin{tabular}{|l|l|}
1 & \\
\hline 1 & \\
\hline
\end{tabular}

\begin{tabular}{l|l}
2 & 3 \\
2 & 3
\end{tabular}

\begin{tabular}{l|l|l}
\hline & 5
\end{tabular}

+

\begin{tabular}{l|l}
1 & 2 \\
\hline 1 & \\
\hline 1 & 2 \\
\hline
\end{tabular}

\begin{tabular}{l}
2 \\
\hline 2 \\
\hline 2 \\
2 \\
2 \\
\hline 2
\end{tabular}

\begin{tabular}{|l|l}
2 & 3 \\
\hline & 3 \\
\hline & 3 \\
\hline
\end{tabular}

\begin{tabular}{l|l}
3 & 4 \\
3 & 4
\end{tabular}

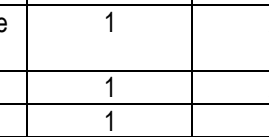

3
3
3
3
3
3

\begin{tabular}{l|l}
3 & \\
\hline 3 & 4 \\
\hline 3 & \\
\hline 3 & \\
\hline 3 &
\end{tabular}

\begin{tabular}{|l|l|}
\hline 4 & \\
\hline 4 & \\
\hline 4 & \\
\hline 4 & \\
\hline 4 & \\
\hline 4 & \\
\hline
\end{tabular}


Questronario

de

Convivenata

1. Hay respeto en la clase:

2. Los alumnos ie relacioran en pequereñas grupas de amiges.

3. Los alumnas se racionan entre fodas

4. Fuera de dase hay retaceen entre las alumnos

5. Los alumnos se ayuctan entre ellos." - - -

6. Hay rivaldad entre las nolas

2. En General, Ta dase eta molivada a trabajar.

8. El favoritrmo de las professies afeda a Tas relación entre los alumnos

4 Existe confianza y libertad con Tastas profercerstas.

- - profesores dedrcan tiempo a habtar con las atomo 10. Los proferorer dedrean hiempo a

11. Hay normas de clase goe se tengan gue cumplis. 12. Hay conflictos entre las alumnos.

13. Hay pardicipación en la clase en genexal - 14. Exiote un orten y organización en la ctabe [detegato, etcr. 15. El grupo enta abrerto a la integración de alumnos nuvevas 16. En general, Tas asigraturas son interesantes.-

17. Existe respetor mutwo eatre el aturnuado y et profesorado.

18. Las problemas se solvcionen parficamenty dralogando?

19. Exmbe rdación entre hodas las atumnes en el recreo 20. Hay confiarize entre los alumnos.

21. En general, existe molivacion por atender las clases

22. Existe un trato respatose entre ras alumiros

23. Se respeta tas datintas firentaciones sexvales:

24. Se respeta las distintas orentaciones eligiosas.

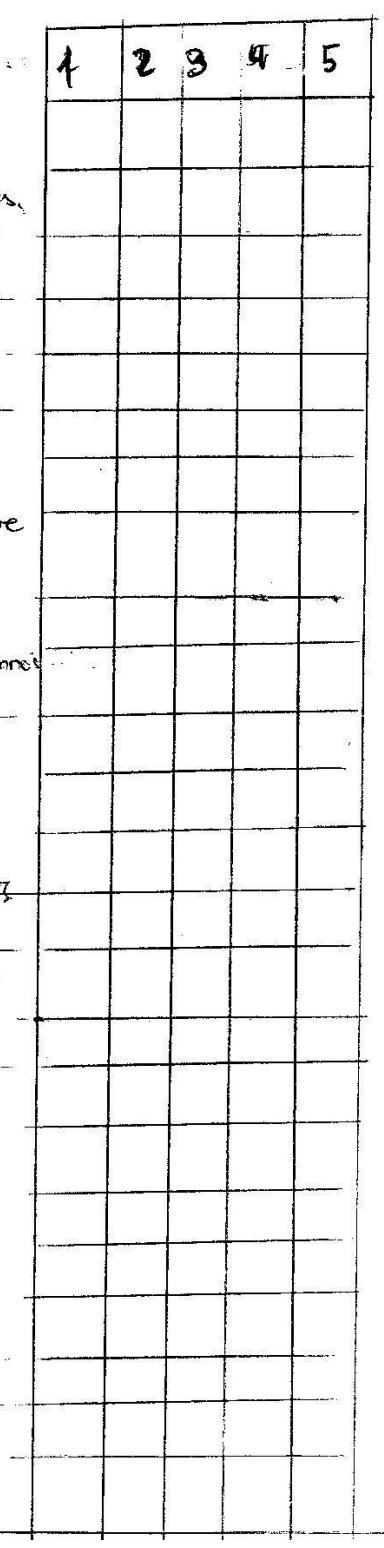




\section{CUESTIONARIO de Convivencia}

1. Existe respesto hacia el profesorado

2. Existe respetc entre los compañeros

$12 \quad 3 \quad 4 \quad 5$

3. Se respetan las orientaciones sexuoles.

$-12345$

4. Afecta

$$
\text { tener parja en Ca clase. }
$$

$2 \quad 3 \quad 4 \quad 5$

5 Afecta al grupo ponerse motes entre los compañeros 12345

6. Existen narmas en clase 12 3. 5

7. Existe acuerdo entre el profesorado y alumnos. 123345

8. El prefesorado debe dar ejemplo a los alumnos.12 2345

9 Se arida el material del centro. $1.2 \quad 3 \quad 4.5$

10. Existe favoritismo por parte del profesorado los alumnos.12345

11. Participan los alumnos en co oreación de las normas 12345

12. Todos los alumnos están integrados en el grupo 12 3 45

13. Existen diperenten grupos en la clase 12 2 345

14. Los alumnos atienden en clase 12345

15. Los alumnos se saltan las clases $12 \quad 3 \quad 4 \quad 5$

16. Los alumnos están motivados $1 \quad 2 \quad 3 \quad 4 \quad 5$

17. los partes mejoran ea convivencia 12345

18. Los partes se corresponden con lo infracián 12345

19. Los alumnos se conden entre si. $12 \quad 3 \quad 4 \quad 5$

20. Las peleas afectan a co convivencia 12345

21. Los alumnos se prestan cos apuntor y los deberes 12345

22. Se habla mal de los compañeros por whatsapp. 12345

23.20 alumnos se defienden ente ellos 12345

24 Los profesores se implican en los problemas de los alumnos 1.2345

25. Se critica a los compañeros a la espalda: 12345

26. Al delegado representa adecuadamente a ca clase 12345 
1 Escuchas al profesor cuando está dando la explicación. 12345

2 Te sientes cómodo hablando en publico en clase. 12345

3 He llevo bien con todos mis compañeros. 12345

4 Discrimino a mis compañeros por su fisico. 12345

5 Molesto a mis compañeros lanzándoles bolitas de papel. 12345

6 La profesora me ignora porque está leyendo los whatsapps:123.345

7 Te divierte que te pegeu cuaudo es de broma 12345

8 Cuaudo no esta el prafesor nos abborotaunas 12345

9 Cuido el material 12345

10. Cuando un campañero hasla do escuchamos 12345

11. En dase estauros dividos por grupos 12345

12. Respetanos ideas de los cern pañeros cuande hacenes trabajo en grupo. $12345 \quad 12345 \%$ 13. Dejannos mustro trabajo para molestar a les demeís. 14. Tenemos reglas en clase 12345

15. Llego tacde a clase. 12345

16. Profesores que trabajom pow y exigen mucho 12345 
17. Me abarro en alqunas clases. 12345 18. Alqunos compañeros insultan cuande sace mejor nota que elles. 12345

19. Me infado chando sacan mais notas que yo.12345 20. Me molesta que mis compañeros cotilleen o we critiquen a la espelden 12345 21. Alqumos profesores tienen preferencias. 12345 22. No hago caso a los projesores. 12345 23. Tenge profesores que, no permiten uader. 12345 24. Tengo compeñeros que me hacen bulling. 12345 25. Cnando alguien me nolestar me chibo al prefesor 26 Nos ayudamas con les deberes. 27. No we qusta que castiguen a tocles por culpa de una persona.

28. No we qusta que ducoufundom con otro che mis compeñseros.

29. Los que tienen buena fana nunca son castigaders. 30. The molesta que cuando preste el material me lo estropeen. o no me lo devneluan. 
1. Ca relació con mis compañers es buena. 12345

2. Mi relación con br profersores es la adecuade. 12345

3. Mi rivel de indivación es elevado. 12345

4. No hay problemas en 6 clase. 12344

S. No hay armbiente tenso en mi clafe. $/ 2345$

6. Tengo confianta con mis compañeros., 2345

7. Ca elase no copia en lor examener., 2345

8. Existe un buen orden en clase. $1234 \mathrm{~S}$

Q. No hay ieracquía entre compañeros. I 23 a $S$

0. Se cumplen las normar de clave., $234 /$

11. Las alamnor tienen interes en ilus califiracioes. 123 is

i2. La clase presta atención al protesorado. 123 \& 5

B. El proteror confía en el alcumnado. 12345

14. Cos alumno solucionan bo problemor habtando. 12345

is. No existe discrimación en el aula. $\quad 2345$

16. Ler proferorer nor guardan respeto. 12345

17. Mantenermer el silencio en clase. 12345

18. Atos) Cos profesores no guardau respeto ni interés por norotros. 12345

10. En clare nos ayudams mutuamente. ( 2345

20. Cos proterores de prácticar nor ayudau. 123445

21. El aubiente en el vecreo no er tenso. 12345

22. Eu la Gibioteca no gritamor. I 23 4 5

23. Asistimas a clase de manera continua. 123 4 5

24. La oritacion sexual en clase us noer causse ningún probleme. 12345

25. Guardesnor orden eh el parillo. 12345

26. Respetamar el moterial escodiar. ( 23 i 5

27. Tracunor lor deberes con regularidad 1234 4

28. Hay alboroto en clase. $\quad 2 \quad 3$ a 5

2a. Participamos en dase. $\quad 234 \mathrm{~s}$

30. Mi clase tene muchos materrotes 12345 